\title{
Was ist Phytotherapie?
}

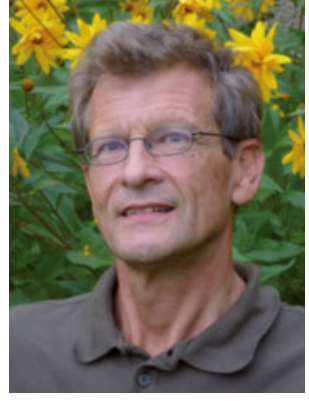

Teilweise gehen die Meinungen über die Inhalte der Phytotherapie recht weit auseinander: Die Definition der Phytotherapie seitens der Österreichischen Gesellschaft für Phytotherapie sieht ausschließlich die Therapie mit Phytopharmaka vor. Demnach hätten wir uns vielleicht ausschließlich mit zugelassenen pflanzlichen Arzneimitteln zu beschäftigen und nicht etwa mit pflanzlichen Nahrungsmitteln oder Nahrungsergänzungsmitteln. Wie steht es aber bezüglich der präklinischen und klinischen Forschung von noch nicht in Arzneimitteln verwendeten Pflanzen, die vielleicht erst in ferner Zukunft eine Arzneimittelzulassung bekommen? Wie steht es mit Pflanzen in Medizinprodukten und Körperpflegemitteln?

Eine Einengung auf nur als Arzneimittel zugelassene Pflanzen und pflanzliche Produkte würde unseren Bereich doch sehr einengen. Der Verbraucher bzw. Patient kümmert sich recht wenig um formale Einstufungen und staatliche Vorschriften, wenn es um den eigenen Gebrauch von Heilpflanzen geht - dies hat übrigens bereits viele Jahrhunderte Tradition!

Überdies sind die gegenwärtigen Einstufungen das Ergebnis von nicht nur fachlich begründeten Prozessen und Entscheidungen, sondern auch von massiven politischen Einflüssen. So manches Phytopharmakon kann sich noch im Prinzip auf der Basis einer positiven Monografie der Kommission E aus den 80er-Jahren „halten“. Aktuelle klinische Belege zur klinischen Evidenz sind mitunter selbst für umsatzstarke Präparate spärlich auch wenn deren Hersteller sich als „forschende Unternehmen“ ausloben.

Das nächste Problem bei der Fokussierung auf pflanzliche Arzneimittel sind die traditionellen Arzneimittel. Für sie wurde ja in der Regel kein Wirksamkeitsnachweis erbracht, und es ist mitunter wegen erheblicher Unterdosierung im Vergleich zu den Dosierungsempfehlungen der Monografien eher zweifelhaft, dass sie einen messbaren Nutzen für die Gesundheit haben. Damit stehen sie recht nahe an Nahrungsmitteln, werden mitunter von solchen übertroffen. Das Kriterium der 30-jährigen Marktpräsenz für traditionelle Arzneimittel ist ursprünglich in Deutschland eine pragmatische politische Härtefallregelung gewesen. Ich hätte damals nicht gedacht, dass dies auf EU-Niveau gehoben wird und dann sogar aus Asien eingeführte Präparate Zeiten für ihren illegalen Marktzutritt angerechnet bekommen. Lesen Sie in diesem Heft den Beitrag von Jütte et al. mit Überlegungen, wie man über diese simplen Pragmasien hinaus eine traditionelle Anwendung tiefgreifender behandeln könnte.

Ich meine also, dass die 3 Gruppen „richtige“ pflanzliche Arzneimittel, traditionelle pflanzliche Arzneimittel und pflanzliche Nahrungs(ergänzungs)mittel jede in sich weit gefächert sind und die Grenzen sich erheblich überlappen bezüglich eines wahrscheinlichen oder eines nachgewiesenen Nutzens für Patienten. Etliche Zubereitungen wechseln auch die Gruppe, zuletzt der ehemalige Star der „rationalen“ Phytotherapie, ein Weißdornextrakt. Er rutschte nach einer misslungenen klinischen Studie mit viel zu hohen Erwartungen in die Gruppe der traditionellen Arzneimittel. Er ist trotzdem genauso nützlich wie davor und dürfte nunmehr das am besten klinisch untersuchte traditionelle Arzneimittel darstellen, auch wenn die meisten Studien inzwischen vergleichsweise alt sind. 
Als traditionelles AM wird nun dieser Weißdornextrakt emsig bei Verbrauchern beworben: „... Mehr Kraft fürs Leben“. Eine solch vage und positive Formulierung hätten allerdings selbst die damaligen und gewiss nicht phyto-feindlichen Mitglieder der Kommission E nicht einmal in Erwägung gezogen. Anderseits kann man konstruieren, dass ein MagenDarm-Mittel „mehr Kraft fürs Leben“ bringen könnte (wenn man mal von quälenden Stuhlproblemen befreit wäre) und erst recht ein Potenzmittel für die älteren Männer (wenn man Sex als zum Leben wichtig erachtet). Ob es womöglich neuere Studien mit Weißdorn zur Steigerung der „Kraft fürs Leben“ gibt, ist mir nicht bekannt; aus den alten fällt mir eine solche Interpretation doch eher schwer, zumal damals die Lebensqualität nie ein
Hauptzielparameter dieser z.T. 30 Jahre alten Studien gewesen ist.

Ja, hier haben wir sie noch einmal, die ominösen 30 Jahre. Es ist eine Generationszeit. Es ist die Zeit, in der sich nach der Wissenschaftstheorie von Fleck und Kuhn neue dogmatische Festlegungen und Konventionen in Fachgebieten bilden können - eigentlich ganz klar: wenn die alten „Chefs“ erst mal weg sind, kommen die jungen ... Die klinische Forschung in medizinischen Randfächern war allerdings vor 30 Jahren noch deutlich rückständig und so war man gegen Bias, Betrug und Fälschung weitaus weniger geschützt als heute. (Heute wird dies allerdings kompensiert durch dreiste und irreführende Publikationen, wie ich beim genaueren Durcharbeiten von Veröffent- lichungen aus allen möglichen Fachzeitschriften für die Rubrik „Forschung kompakt“ recht häufig feststellen muss.) Daher wäre es schön, wenn die hochdosierten traditionellen Arzneimittel ihren Nutzen durch neuere Studien bestätigen - auch wenn dies vom Gesetz nicht vorgesehen ist.

Es gibt ja sogar Lebensmittel und Körperpflegemittel mit medizinischem Nutzen, der durch aussagekräftige klinische Studien belegt ist. Sollen wir solche aus formalen Gründen einer zu engen Definition der Phytotherapie ignorieren? $\mathrm{Zu}$ letzt: Es gibt auch Präparate, die völlig identisch im einen Land als Lebensmittel und im anderen als Arzneimittel angeboten werden ...

Bernhard Uehleke 